\title{
Effectiveness of Propolis, Probiotics And Chlorhexidine on Streptococcus Mutans And Candida Albicans: An In-Vitro Study.
}

\author{
Dr. Rajani Mary George ${ }^{1}$,Dr. Akash V. Kasliwal ${ }^{2}$ \\ ${ }^{I}$ (Reader, Department Of Public Health Dentistry, A. J Institute Of Dental Sciences, Mangalore, \\ Karnataka, India) \\ ${ }^{2}$ (Post Graduate Student, Department Of Public Health Dentistry, A. J Institute Of Dental Sciences, \\ Mangalore, Karnataka, India)
}

\begin{abstract}
Introduction: Natural products have always been used by the health industries as alternatives to the conventional allopathic formulations for prevention and treatment of various health problems. Flavonoids, present in propolis obtained from honey bees have antibacterial, antifungal and anti-inflammatory proprieties. Probiotics are food products containing beneficial micro-organisms, which stimulate health promoting flora thus, suppressing the pathologic colonization and disease spread.

Objective: To determine and compare the effectiveness of propolis, probiotics and chlorhexidine on Streptococcus mutans and Candida albicans.

Methods: An in vitro study was conducted to test the effectiveness of propolis, probiotics and chlorhexidine on $S$ mutans and $C$ albicans. The antimicrobial activity was determined using Agar Diffusion Technique-Well method. Equidistant wells were bored into Muller Hinton Agar plates using a cork borer and then filled with 50 $\mu l$ of each of the test products. These plates were then left to dry at room temperature for 2 hours and then incubated at $37^{\circ} \mathrm{C}$ for 24 hours and examined for the zone of inhibition.

Results: The mean zone of inhibition for $S$ mutans was maximum for propolis $(14.6 \mathrm{~mm})$ and minimum for probiotics $(9.4 \mathrm{~mm})$. Similarly, for C albicans the values obtained were 15.6mm, $12 \mathrm{~mm}$ and $14 \mathrm{~mm}$ for propolis, probiotics and chlorhexidine respectively.

Conclusion: Propolis is as good as chlorhexidine in inhibiting $S$ mutans and better than chlorhexidine in inhibiting $C$ albicans in-vitro; whereas propolis inhibits both the organisms better than probiotics.
\end{abstract}

Keywords: Caries, Natural products, Oral Microorganisms, Probiotics, Propolis

\section{Introduction}

Natural products have always been used by the health industries as alternatives to the conventional allopathic formulations for prevention and treatment of various health problems. Apitherapy, or "bee therapy" is the medicinal use of products made by honeybees.[1] Propolis is a resinous yellow brown to dark brown, sticky substance that honey bees (Apis mellifera) collect from tree buds, sap flows, shrubs or other botanical sources.[2]

Propolis is composed of highly active bio-chemical substances known as bioflavenoids (Vitamin P). It also comprises of resins and balsams, essential oils and wax, pollens and small amounts of amino acids, minerals, vitamin A, B-complex and E. ${ }^{[2]}$ Flavonoids, which are one of the main chemical components of propolis; are well known plant compounds that have antibacterial, antifungal, antiviral, antioxidant and anti-inflammatory properties.[2]

Probiotics are dietary supplements which have been advocated for the prevention and the treatment of a wide range of diseases. The beneficial micro-organisms present in these products suppress the spread of disease and pathological colonization by stimulation of health promoting flora.[3] The most commonly used probiotic bacterial strains belong to the genera Lactobacillus and Bifidobacterium.[4] Species commonly isolated from saliva samples include L. paracasei, L. plantarum, L. rhamnosus, and L. salivarius. [4] A good probiotic agent needs to be non-pathogenic, nontoxic, resistant to gastric acid, adhere to gut epithelial tissue and produce antibacterial substances.[3]

Traditionally, probiotics have been associated with gastrointestinal health. The prevention and treatment of GIT infections and diseases have been the focus of interest for clinical use of probiotics. It has also been found useful for the enhancement of adaptive immunity, treatment and prevention of respiratory tract infection, alleviation of allergies and treatment of ailments like atopic dermatitis, asthma and rhinitis. [4] However, recent reviews have reported on the use of probiotic strains for the prevention of oral diseases, including caries and periodontal diseases.[4, 5]

Chlorhexidine gluconate is a cationic bisbiguanide which is effective against an array of microorganisms, including gram positive and gram negative organisms, fungi, yeasts and viruses. Chlorhexidine 
exhibits both anti plaque and antibacterial properties and is considered as a gold standard in the treatment and prevention of oral diseases, especially periodontal diseases.[6] The present study will compare the effect of propolis and probiotics on Streptococcus mutans and Candida albicans.

\section{Materials And Methods}

The microbiological testing was carried out in Mangalore Biotech Laboratory, Mangalore; in August 2015. Propolis extract prepared from the crude propolis was procured from Bangalore Herbal Suppliers. Commercially available probiotic capsules containing Lactobacillus sporongenes (50 million), Streptococcus fecalis (30 million) and Clostridium butricum (2 million); and chlorhexidine mouthwash were obtained from local medical store in the city. The antimicrobial activity was determined using Agar Diffusion Technique-Well method. $^{[7]}$

Fresh cultures of the test micro-organisms namely, Streptococcus mutans and Candida albicans were revived from $-80^{\circ} \mathrm{C}$ and inoculated into freshly prepared Tryptic Soy Broth (TS Broth). These inoculated broths were then incubated at $37^{\circ} \mathrm{C}$ for 24 hours. After incubation $S$ mutans culture was streaked into Mitis Salivarius Agar plates whereas $C$ albicans culture was streaked into Hichrome Candida Agar plates. These plates were again incubated at $37^{\circ} \mathrm{C}$ for 36 hours; this led to the growth of final culture of the micro-organisms that were to be tested. The cultures of micro-organisms were then spread separately on Muller Hinton Agar (MHA) plates. Equidistant wells of $5 \mathrm{~mm}$ diameter and 1-2mm dept were bored into the Muller Hinton Agar plates using a sterile cork borer.

The powder from probiotic capsule was mixed with $2 \mathrm{ml}$ distilled water to achieve desired consistency. Propolis extract and chlorhexidine were already available as liquid solutions. The wells on MHA plates were then filled with $50 \mu \mathrm{l}$ of the test products i.e propolis extract, probiotic solution and chlorhexidine solution. These plates were then left to dry at room temperature for 2 hours and then incubated at $37^{\circ} \mathrm{C}$ for 24 hours. Five such samples of each product were made and the antimicrobial activity was determined by measuring the diameter of the zone of inhibition of each of the product in millimeters. All the samples were made in duplicate and the experiment was repeated once again to minimize the error. The evaluation of the plates was performed by two independent observers, who were blinded with regards to the products used. In case of disagreement a consensus was reached after discussion. The data was processed in SPSS software version 17. Mann-Whitney U test and Kruskal-Wallis test were applied. A p value of $<0.05$ was considered as statistically significant.

\section{Results}

The results of antimicrobial growth inhibition are summarized in Table 1. For $S$ mutans the mean zone of inhibition was maximum for propolis $(14.6 \mathrm{~mm})$ and minimum for probiotics $(9.4 \mathrm{~mm})$. Similarly, for $C$ albicans the values obtained were $15.6 \mathrm{~mm}, 12 \mathrm{~mm}$ and $14 \mathrm{~mm}$ for propolis, probiotics and chlorhexidine respectively.

On intergroup comparison (Table 2) all results were statistically significant. Propolis was highly effective against $S$ mutans and $C$ albicans when compared with chlorhexidine. Probiotics had weak antimicrobial activity against both micro organisms in comparison with chlorhexidine and propolis.

\section{Discussion}

Most oral diseases are primarily caused, or at least modified, by bacteria that inhabit the oral cavity.[7] This study was conducted on $S$ mutans and $C$ albicans because the former is the major causative organism of most prevalent dental disease namely dental caries; whereas the latter is a commonly encountered organism in both oral and systemic fungal infections. To obtain and maintain optimum oral health, the prophylactic and therapeutic interventions have always aimed to reduce the microbial load of the oral cavity.[7] Use of natural products containing antimicrobial properties support these efforts by inhibiting the growth of oral pathogenic micro-organisms or by aiding in removal of established biofilm.[7]

Propolis exhibits antimicrobial, anti-inflammatory, healing, anesthetic and cariostatic properties.[8, 9] It prevents fungal cell division and also breaks down fungal cell wall and cytoplasm similar to the action of some antibiotics.[9]

In the present study propolis showed the highest in vitro inhibitory effect against $\mathrm{S}$ mutans followed by chlorhexidine and then probiotics. In a study done by Steinberg D et al., Propolis demonstrated an antibacterial effect both in vitro on isolated oral streptococci and in the clinical study on salivary bacterial counts.[10] In a review by Waldner-Tomic N.M et al., on the in vitro Antimicrobial Efficacy of Propolis against Four Oral Pathogens; they found that for $S$ mutans, the required mean minimum bactericidal concentration was almost 100-times higher than that of the control group. The control substance in this case was chlorhexidine, vestitol and neovestitol. Whereas the result of studies assessing the minimum inhibitory concentration is provided that the effect of propolis was lower than the control substances, especially when $S$ mutans and $P$ gingivalis were tested. The minimum inhibitory concentration for $S$ mutans was comparable to the ones obtained by the 
corresponding control groups.[7] Another study done by Ivanvajic S et al., shows that propolis has antimicrobial activity not only against streptococci but also against a variety of other gram positive and gram negative bacteria. The results of all these studies are in concurrence with the present study.[11]

According to the results of the present study, the zone of inhibition for $C$ albicans was highest for propolis followed by chlorehexidine and probiotics respectively. In an in-vitro study done by SP Tyagi et al., Comparing of antimicrobial efficacy of propolis, Morinda citrifolia, Azadirachta indica (Neem) and 5\% sodium hypochlorite on $C$ albicans biofilm formed on tooth substrate, in-vitro, showed that Sodium hypochlorite and propolis groups exhibited highest antimicrobial efficacy against $C$ albicans with no statistically significant difference.[9] In another study done by Arslan S., et al propolis and $\mathrm{NaOCl}$ were more effective against $C$ albicans than chlorhexidine and Mineral Trioxide Aggregate; which is similar to the results of the present study.[12]

Possible actions of probiotic bacteria in the oral environment are competition of binding sites, production of antimicrobial substances and activation and regulation of the immune response. Bacterial antagonism may occur when growth of one bacterial species is hampered by components produced by another species.[13] Though the predominant strategy for caries prevention relies on influencing the re- and demineralization processes, mainly by using fluorides but also other factors involved in the caries process may be targeted; one alternative could be to promote colonization of caries inhibiting bacteria and in this aspect, probiotic bacteria constitute a novel concept.[13] Also because they are easily available in dairy products, fruit drinks drops, gruels, chewing gums and tablets in the market.

In the present study, probiotics showed the least antimicrobial activity against $S$ mutans. In a study done by Hasslöf et al., the selected probiotic strains showed a significant but somewhat varying ability to inhibit growth of oral mutans streptococci.[13] The study was conducted on 8 strains of probiotic organisms out of which all lactobacilli strains inhibited the growth of the $S$ mutans completely with the exception of $L$ acidophilus La5. L acidophilus La5 had a statistically significantly weaker inhibition capacity in comparison with the other probiotic strains. In the present study L sporogenes was the chief probiotic organism present in the capsule this would be the reason for the weak inhibition of S. mutans by probiotic.

In study conducted by Jothika $\mathrm{M}$ et al., to determine the short-term efficiency of probiotic, chlorhexidine, and fluoride mouthwashes on plaque Streptococcus mutans level at four periodic intervals; they found that Chlorhexidine, sodium fluoride, and probiotic mouthwashes reduce plaque $S$ mutans levels. Probiotic mouthwash is effective and equivalent to chlorhexidine and sodium fluoride mouthwashes.[14] The difference in the results in present study could be due to the type of probiotic bacteria present in the capsules used in this study as different strains of probiotic organisms have different effectiveness. In a review article by Gomes $\mathrm{R}$ et al., it was stated that for the probiotic to be able to exert an anticariogenic effect; primarily the bacteria must be capable of adhering to the dental surface; second, it must become part of the biofilm; and finally, it must compete with cariogenic bacteria reducing the level of colonization of these.[15]

The inhibition of $C$ albicans by probiotic in the present study was least as compared to propolis and chlorhexidine. In the study done by Hasslöf et al., it was seen that all the tested lactobacilli strains reduced candida growth but the effect was generally weaker than for mutans streptococci which is in concurrence with the present study.[13]

\section{Tables}

TABLE 1: Mean Zone Of Inhibition of The Test Groups

\begin{tabular}{|l|l|l|}
\hline $\begin{array}{c}\text { Microbes } \\
\text { Groups }\end{array}$ & $\begin{array}{l}\text { S Mutans } \\
\text { (Mean in } \mathrm{mm} \text { ) }\end{array}$ & $\begin{array}{l}\text { C Albicans } \\
\text { (Mean in mm) }\end{array}$ \\
\hline Propolis & 14.6 & 15.6 \\
\hline Probiotics & 9.4 & 12.00 \\
\hline Chlorhexidine & 14.00 & 14.00 \\
\hline
\end{tabular}

Table 2: Comparison of test groups with regards to their antimicrobial effectiveness

\begin{tabular}{|l|l|l|l|l|}
\hline \multirow{2}{*}{ Test Groups } & \multicolumn{4}{|c|}{ Micro organisms } \\
\cline { 2 - 5 } & \multicolumn{2}{|c|}{ S mutans } & C albicans \\
\cline { 2 - 5 } & Z value & p value & Z value & p value \\
\hline Propolisv/s Probiotics & -2.694 & 0.007 & -2.835 & 0.005 \\
\hline $\begin{array}{l}\text { Propolisv/s } \\
\text { Chlorhexidine }\end{array}$ & -1.964 & 0.050 & -2.835 & 0.005 \\
\hline $\begin{array}{l}\text { Probioticsv/s } \\
\text { Chlorhexidine }\end{array}$ & 2.895 & 0.005 & -3.000 & 0.003 \\
\hline
\end{tabular}




\section{Conclusion}

As we are living in an era of evidence based medicine, any material with potential clinical application must go through a series of tests to demonstrate biocompatibility to the tissues of oral cavity as well as marked advantages in terms of effectiveness in maintaining oral health and preventing oral diseases. According to the present study it can be concluded that propolis is as good as chlorhexidine in inhibiting $S$ mutans and significantly better than probiotics in inhibiting $C$ albicans; whereas probiotics is inferior to both propolis and chlorhexidine in its effectiveness against the same. However, in vivo studies will also be required for recommending ideal clinical protocols using these products

\section{References}

[1]. Ahuja V, Ahuja A. Apitherapy - A sweet approach to dental diseases. Part II: Propolis. J Academy Adv Dent Res 2011; 2(2): 2-8.

[2]. Parolia A, Thomas MS, Kundabala M and Mohan M. Propolis and its potential uses in oral health. Int. J. Med. Med. Sci 2010; 2(7): 210-15.

[3]. Chopra R, Mathur S. Probiotics in dentistry: A boon or sham. Dent Res J (Isfahan) 2014; 11(3): 308-15

[4]. Haukioja A. Probiotics and Oral Health. Eur J Dent 2010; 4(3): 348-55.

[5]. Cagetti M G, Mastroberardino S, Milia E, Cocco F, Lingström P, and Campus G. The Use of Probiotic Strains in Caries Prevention: A Systematic Review. Nutrients 2013; 5(7): 2530-50.

[6]. Peter S, Essentials of Preventive and Community Dentistry, $4^{\text {th }}$ Edition. Pg. no: 131

[7]. Waldner-Tomic N.M, Vanni R, Belibasakis GN, Thurnheer T, Attin T, Schmidlin PR. The in Vitro Antimicrobial Efficacy of Propolis against Four Oral Pathogens: A Review. Dent. J. 2015, 2: 85-97.

[8]. Hayacibara M.F, Koo H, Rosalen PL, Duarte S, Franco EM, Bowen WH, Ikegaki M, Cury JA. In vitro and in vivo effects of isolated fractions of Brazilian propolis on caries development. J Ethnopharm 2005; (101):110-15.

[9]. Tyagi SP, Sinha DJ, Garg P, Singh UP, Mishra CC, Nagpal R. Comparison of antimicrobial efficacy of propolis, Morinda citrifolia, Azadirachta indica (Neem) and 5\% sodium hypochlorite on Candida albicans biofilm formed on tooth substrate: An in-vitro study. $\mathbf{J}$ Conserv Dent 2013; 16(6): 532-35.

[10]. Steinberg D, Kaine G, Gedalia I. Antibacterial effect of propolis and honey on oral bacteria. Am. J. Dent 1996; 9(6):236-39.

[11]. Ivančajić S, Mileusnić I, Milošević DC. In vitro antibacterial activity of propolis extracts on 12 different bacteria in conditions of 3 various $\mathrm{pH}$ values. Arch Biol Sci Belgrade 2010; 62: 915-34.

[12]. Arslan S, Ozbilge H, Kaya EG, Ozgur Er. In vitro antimicrobial activity of propolis, BioPure MTAD, sodium hypochlorite, and chlorhexidine on Enterococcus faecalis and Candida albicans. Saudi Med J 2011; 32 (5): 479-83

[13]. Hasslöf P, Hedberg M, Twetman S, Stecksén-Blicks C. Growth inhibition of oral mutans streptococci and candida by commercial probiotic lactobacilli - an in vitro study. BMC Oral Health 2010; 10:18

[14]. Jyothika M, Vanajassun PP, Someshwar B. Effectiveness of probiotic, chlorhexidine and fluoride mouthwash against Streptococcus mutans - Randomized, single-blind, in vivo study. J Int Soc Prev Community Dent. 2015 May; 5(Suppl 1):44-8.

[15]. Gomes R, Miyazak M, Filho IJ. Action of probiotics on oral pathogens: Efficacy and Controversies. Dent Oral Craniofac Res 2015;1(4): 121-25 